\title{
Latest Developments in the Assessment and Treatment of Lower Urinary Tract Symptoms Suggestive of Benign Prostatic Hyperplasia: What is Clinically Relevant?
}

\author{
Matthias Oelke
}

(C) Springer International Publishing Switzerland 2014

Lower urinary tract symptoms suggestive of benign prostatic hyperplasia (LUTS/BPH) are prevalent and increase with aging. Epidemiological studies have shown that approximately $40 \%$ of men aged $\geq 50$ years have bothersome LUTS/BPH [1]. It is therefore not surprising that LUTS/BPH are positioned at 7th place in the most frequently diagnosed and most costly diseases in men aged $\geq 50$ years [2]. Healthcare-seeking behavior is triggered by LUTS severity and LUTS phenotypes. Men often consult their doctors when they have severe LUTS or experience urinary urgency or nocturia $[1,3]$. LUTS/BPH have a multifactorial etiology and can be caused by the prostate, bladder, urethra, pelvic floor, central or peripheral nervous system, ureters, or even by the kidneys in case of nocturnal polyuria [4]. LUTS can be divided into storage (e.g., urgency, frequency, nocturia), voiding (e.g., hesitancy, poor stream, intermittency), and post-micturition symptoms (post-void dribbling, feeling of incomplete bladder emptying) [5].

The majority of guidelines on LUTS/BPH have been established by urologists [4, 6, 7] but can also be-more or less easily-implemented by other medical specialties. Different healthcare systems worldwide have different regulations and defined responsibilities regarding which medical specialty should primarily assess and treat men with LUTS/BPH. While almost three-quarters of men with LUTS/BPH in Germany are diagnosed and treated by urologists [8], the majority of men in other countries, for example Australia or the UK, are managed by primary-care physicians. Specialized training programs and experience

M. Oelke $(\square)$

Department of Urology, OE 6240, Hannover Medical School, 30625 Hannover, Germany

e-mail: oelke.matthias@mh-hannover.de with patients with LUTS/BPH are therefore mandatory for different medical specialties.

Careful assessment helps to identify the underlying pathophysiology of LUTS. Simple patient history or a symptom questionnaire cannot distinguish between LUTS of benign or malignant origin because the type of symptom or symptom severity is nonspecific for the underlying disease. This may limit the widespread assessment and treatment of LUTS by non-urologic professionals because special assessment tools-before treatment initiation-are usually not available in the offices of primary-care physicians (e.g., uroflowmeter, transrectal ultrasound, multichannel urodynamics, urethrocystoscopy). It is therefore crucial to know when nonurologists should transfer their patients to specialized urology care. An expert panel recommended specialized urology support in particular when physicians are in doubt of the origin or benign character of LUTS, in cases of macroscopic hematuria, or in men with absolute indications for prostate surgery [9].

Adherence to guidelines is crucial for every medical specialty dealing with LUTS/BPH because real-life practice in the USA has shown that doctors who follow guidelines perform significantly less prostate surgery than doctors who do not [10]. Doctors with poor adherence to guidelines will have performed prostate surgery within the first year of diagnosis in $11 \%$ of patients, whereas doctors with good adherence to guidelines operated on only $2 \%$ of their patients (which translates into a $91 \%$ decrease in the adjusted odds of receiving surgery with good vs. poor guideline adherence). Prostate surgery can relieve LUTS, but approximately $20 \%$ of patients have persisting bothersome symptoms [11]. Longitudinal studies in France in men after prostate surgery have shown that approximately $25 \%$ of patients after 1 year and approximately $40 \%$ of 
patients after 5 years are again using drugs for the treatment of LUTS [12].

When judging the effects of prostatic surgery versus drugs tested in placebo-controlled trials one has to consider that patients always start with a washout period and placebo run-in phase during which LUTS will already have reduced substantially (usually by 2-4 IPSS [International Prostate Symptom Score] points on average) [4]. When adding this initial LUTS (IPSS) reduction to the effects of the treatment period, $\alpha_{1}$-adrenoceptor antagonists $(\alpha$ blockers) almost equally reduce LUTS compared to surgery. For example, the new $\alpha$-blocker silodosin has shown an average LUTS reduction of 7 IPSS points in 12-week phase III trials, but this reduction has been approximately 9-11 IPSS points when comparing LUTS at study start with LUTS at study end [13].

$\alpha$-Blockers have become the most popular drug class for the treatment of LUTS/BPH over the past 25 years. Primary candidates for $\alpha$-blocker therapy are men with moderate-to-severe bothersome voiding or voiding + storage LUTS [4]. The reasons for this first-line treatment choice are obvious [4]: $\alpha$-blockers can be administered in the majority of patients with LUTS/BPH because the majority of men suffer voiding or voiding + storage symptoms; $\alpha$-blockers are already efficacious after some days of treatment; they significantly and substantially reduce LUTS (IPSS) within weeks (decrease in IPSS by $30-40 \%$ on average after placebo run-in and up to $50 \%$ in open-label trials); significantly improve urinary flow rate within hours (by 20-25\%); significantly improve health-related quality of life; have a long-lasting effect on symptoms, thereby reducing symptomatic disease progression; and work independently of the patient's age, prostate volume, and initial symptom severity. However, $\alpha-$ blockers cannot prevent (acute) urinary retention or need for prostatec surgery in long-term studies, especially in patients with enlarged prostates at baseline $(>40 \mathrm{cc}$ ) [14].

All $\alpha$-blockers used in Europe have proven efficacy in reducing LUTS/BPH in randomized short-term ( $\leq 3$ months) and long-term trials ( $\geq 12$ months) [4]. Additionally, all $\alpha$-blockers are equally effective despite differences in $\alpha_{1}$-adrenoceptor subtype inhibition. However, the adverse event profiles of the commonly used $\alpha$-blockers in Europe are substantially different. Therefore, patients have to be carefully evaluated first (including the analysis of currently used prescriptions and over the counter drugs), the $\alpha$-blocker should be chosen according to existing co-morbidities, and patients should be counselled with regard to the expected adverse event profile. Important limitations of individual $\alpha$-blockers are treatment-related cardiovascular, sexual, and ocular adverse events.

Orthostatic hypotension, dizziness, and asthenia are a great concern for many $\alpha$-blockers, especially for those that are nonspecific for $\alpha_{1 \mathrm{~A}^{-}}, \alpha_{1 \mathrm{~B}^{-}}$, and $\alpha_{1 \mathrm{D}^{-}}$-adrenoceptor subtypes and administered as immediate-release formulations [15]. $\alpha_{1 \mathrm{~B}}$-Adrenoceptors are primarily found in blood vessels and inhibition of these receptors by nonspecific $\alpha_{1}$-blockers are most likely responsible for a decrease in blood pressure during treatment. $\alpha$-Blockers as a class have originally been developed for the treatment of arterial hypertension. Doxazosin and terazosin are nonspecific for $\alpha_{1}$-adrenoceptor subtypes and lower blood pressure significantly, especially upon treatment initiation ("first dose hypotension"); consequently, these $\alpha$-blockers have to be dose-titrated when immediate-release formulations are used. The extendedrelease gastro-intestinal therapeutic system (GITS) formulations of doxazosin and alfuzosin have a better tolerability profile but still lower blood pressure significantly [16]. In contrast, tamsulosin has a rather high selectivity for $\alpha_{1 \mathrm{~A}^{-}}$ adrenoceptors and reduces blood pressure less frequently and in a less pronounced way. Nevertheless, in a recently published study even tamsulosin had significantly higher rates of severe hypotension requiring hospital admissions during the first two treatment months when compared to $5 \alpha$ reductase inhibitors, a drug class without relevant effects on blood pressure [17]. The hazard ratio for severe hypotension was 2.12 (95\% CI 1.29-3.04) for the first month and 1.51 (95\% CI 1.04-2.18) for the second month. In contrast, the new $\alpha$-blocker silodosin is highly specific for $\alpha_{1 \mathrm{~A}}$-adrenoceptors and does not significantly affect blood pressure, as shown in phase III trials [13]. This is a clear advantage for the treatment of male LUTS especially for men who are sensitive to a decrease in blood pressure as the prescribing physician or the patient does not need to dose-titrate or take precautions against undesired cardiovascular adverse events.

$\alpha$-Blockers in general do not have adverse effects on libido or erectile function but may have effects on ejaculation [15]. It was long believed that ejaculation disorders (reduced semen volume or dry ejaculation) were caused by retrograde ejaculation but recent studies have demonstrated that $\alpha$-blockers cause (relative) anejaculation as no semen or sperm cells were detected in the bladder after ejaculation [18]. Of all available $\alpha$-blockers and formulations, only tamsulosin and silodosin have consistently shown increased rates of anejaculation, which can occur in up to $28 \%$ of patients treated with silodosin $8 \mathrm{mg}$ once daily $[13,15]$. However, post hoc analyses with silodosin have demonstrated that anejaculation was often associated with a more pronounced LUTS reduction and high treatment persistence [19]. Anejaculation is no special threat and reversible but the patient needs to be counselled accordingly.

Although $\alpha$-blockers have been thoroughly investigated in long-term trials and used for many years in clinical practice, ocular adverse events first became evident in 2005 when three phenomena were observed during cataract surgery in tamsulosin users: (1) a floppy iris that flutters 
and billows according to normal intraoperative fluid movements, (2) prolapse of iris tissue with surgical incisions, and (3) progressive intraoperative miosis despite standard preoperative pupil dilation. This triad is now known as the "intraoperative floppy iris syndrome," or IFIS, but, since it was first mentioned, has been described for almost all $\alpha$-blockers for the treatment of LUTS or arterial hypertension (including prazosin, indoramin, and labetalol) and, interestingly, also for drugs with partial $\alpha$ adrenoceptor blocking abilities (e.g., antipsychotics, antidepressants, benzodiazepines, serenoa repens [saw palmetto]) or even finasteride [15]. IFIS can occur in one eye only, may be incomplete (appearance of only one or two phenomena of the triad), and can be seen in both men and women. It is assumed that IFIS is irreversible. Preliminary results in experimental animals suggest that IFIS results from pharmacologic inhibition of the smooth musculature of the iris and smooth muscle atrophy due to drug accumulation in the pigment of the iris. Although IFIS is not life threatening, precautions should be taken in patients who are scheduled for cataract surgery, such as delaying $\alpha$ blocker use, prescribing PDE5-inhibitors instead of $\alpha$ blockers (for an overview see Ückert and Oelke [20]), and having only experienced ophthalmologists performing cataract surgery (for an overview and recommendations see the American Academy of Ophthalmology [15]). In contrast to reports on IFIS after the intake of the above-mentioned $\alpha$-blockers, no reports have yet been published on IFIS in patients with silodosin. It remains to be determined whether this reflects the absence of this particular adverse event or the late introduction of this drug into the market with fewer patients exposed to silodosin.

In the context of clinical practice, guidelines, and $\alpha$ blockers as first-line treatment of LUTS/BPH I am happy to present this supplement issue of Clinical Drug Investigation. The first article is dedicated to primary-care physicians' patterns of practice assessment of men with LUTS/ BPH in Portugal [21]. This article adds valuable information regarding the characteristics of men who seek medical help for LUTS, to the diagnostic approach, and on factors that trigger referrals to urologists. It is evident that assessment of LUTS/BPH does not differ much in primary care compared to in specialized urology care. I am pleased to read that primary-care physicians tend to seek urology support when patients have severe LUTS (IPSS $\geq 20$ ), large prostate glands, voiding symptoms suggestive of benign prostatic obstruction, and insufficient treatment response during initial therapy, and when physicians have little experience with LUTS/BPH patients. The common assessment practices in Portugal therefore appear to be compatible with the recommendations formulated by urologists in 2012, who defined the circumstances under which patients should be referred for specialized care [9].
The second article deals with the first-line treatment of male LUTS, the $\alpha$-blockers [22]. This drug class is especially attractive for primary care and especially when there are no absolute indications for prostate surgery. Julio Fonseca and Carlos Martins da Silva clearly and nicely list the characteristics, advantages, and disadvantages of all available $\alpha$-blockers in Europe. Therefore, this review article will be beneficial for all professionals who are involved in the treatment of male LUTS. There is a particular focus on the new $\alpha$-blocker silodosin, which has been shown to have advantages over other $\alpha$-blockers, especially when hypotension, dizziness, asthenia, or IFIS are consideration in the treatment of LUTS/BPH.

Conflict of interest Matthias Oelke is a lecturer, advisor and/or study participant in the field of LUTS/BPH for Apogepha, Astellas, GlaxoSmithKline, Lilly, Mundipharma, Pfizer, and Recordati.

\section{References}

1. Berges RR, Pientka L, Höfner K, Senge T, Jonas U. Male lower urinary tract symptoms and related health care seeking in Germany. Eur Urol. 2001;39:682-7.

2. Fenter TC, Naslund MJ, Shah MB, Eaddy MT, Black L. The cost of treating the 10 most prevalent diseases in men 50 years of age or older. Am J Manag Care. 2006;4(Suppl):S90-8.

3. Oelke M, Wiese B, Berges R. Nocturia and its impact on health related quality of life and health care seeking behaviour in German community-dwelling men aged 50 years or older. World J Urol. 2014;32:1155-62.

4. Oelke M, Bachmann A, Descazeaud A, Emberton M, Gravas S, Michel MC, et al. EAU Guidelines on the treatment and follow-up of non-neurogenic male lower urinary tract symptoms, including benign prostatic obstruction. Eur Urol. 2013;64:118-40.

5. Abrams P, Cardozo L, Fall M, Griffiths D, Rosier P, Ulmsten U, et al. The standardisation of terminology of lower urinary tract function: report from the Standardisation Sub-committee of the International Continence Society. Neurourol Urodyn. 2002;21:167-78.

6. Jones C, Hill J, Chapple C, Guideline Development Group. Management of lower urinary tract symptoms in men: summary of NICE guidance. BMJ. 2010;340:c2354.

7. McVary KT, Roehrborn CG, Avins AL, Barry MJ, Bruskewitz $\mathrm{RC}$, Donnell RC, et al. Update on AUA guideline on the management of benign prostatic hyperplasisa. J Urol. 2011;185:1793-803.

8. Berges RR, Pientka L. Management of the BPH syndrome in Germany: who is treated and how? Eur Urol. 1999;36(Suppl 3):21-7.

9. Oelke M, Burger M, Castro-Diaz D, Chartier-Kastler E, JiménezCidre MA, McNicholas T, et al. Diagnosis and medical treatment of LUTS in adult men: applying specialist guidelines in clinical practice. BJU Int. 2012;110:710-8.

10. Strope SA, Wei JT, Smith A, Wilt TJ, Saigal CS, Elliott SP, Urologic Diseases in America Project. Evaluative care guideline compliance is associated with provision of benign prostatic hyperplasia surgery. Urology. 2012;80:84-9.

11. Seaman EK, Jacobs BZ, Blaivas JG, Kaplan SA. Persistence or recurrence of symptoms after transurethral resection of the prostate: a urodynamic assessment. J Urol. 1994;152:935-7. 
12. Lukacs B, Cornu JN, Aout M, Tessier N, Hodee C, Haab F, et al. Management of lower urinary tract symptoms related to benign prostatic hyperplasia in real-life practice in France: a comprehensive population study. Eur Urol. 2013;64:493-501.

13. Chapple CR, Montorsi F, Tammela TL, Wirth M, Koldewijn E, Fernandez-Fernandez E, European Silodosin Study Group. Silodosin therapy for lower urinary tract symptoms in men with suspected benign prostatic hyperplasia: results of an international, randomized, double-blind, placebo- and active-controlled clinical trial performed in Europe. Eur Urol. 2011;59:342-52.

14. Roehrborn CG, Siami P, Barkin J, Damiao R, Major-Walker K, Nandy I, et al. The effects of combination therapy with dutasteride and tamsulosin on clinical outcomes in men with symptomatic benign prostatic hyperplasia: 4-year results from the CombAT study. Eur Urol. 2010;57:123-31.

15. Oelke M, Gericke A, Michel MC. Cardiovascular and ocular safety of $\alpha_{1}$-adrenoceptor antagonists in the treatment of male lower urinary tract symptoms. Expt Opin Drug Saf. 2014;13:1187-97.

16. Nickel JC, Sander S, Moon TD. A meta-analysis of the vascularrelated safety profile and efficacy of a-adrenergic blockers for symptoms related to benign prostatic hyperplasia. Int J Clin Pract. 2008;62:1547-59.
17. Bird ST, Delaney JA, Brophy JM, Etminan M, Skeldon SC, Hartzema AG. Tamsulosin treatment for benign prostatic hyperplasia and risk of severe hypotension in men aged 40-85 years in the United States: risk window analyses using between and within patient methodology. BMJ. 2013;347:f6320.

18. Hisasue S, Furuya R, Itoh N, et al. Ejaculatory disorder caused by alpha-1 adrenoceptor antagonists is not retrograde ejaculation but a loss of seminal emission. Int J Urol. 2006;13:1311-6.

19. Roehrborn CG, Kaplan SA, Lepor H, Volinn W. Symptomatic and urodynamic responses in patients with reduced or no seminal emission during silodosin treatment for LUTS and BPH. Prostate Cancer Prostatic Dis. 2011;14:143-8.

20. Ückert S, Oelke M. Phosphodiesterase (PDE) inhibitors in the treatment of lower urinary tract dysfunction. Br J Clin Pharmacol. 2011;72:197-204.

21. Fonseca J, da Silva CM. The diagnosis and treatment of lower urinary tract symptoms due to benign prostatic hyperplasia by primary care physicians in Portugal. Clin Drug Invest. 2014. doi:10.1007/s40261-014-0256-4

22. Fonseca J, da Silva CM. The diagnosis and treatment of lower urinary tract symptoms due to benign prostatic hyperplasia with $\alpha$-blockers: focus on silodosin. Clin Drug Invest. 2014. doi:10. 1007/s40261-014-0257-3 